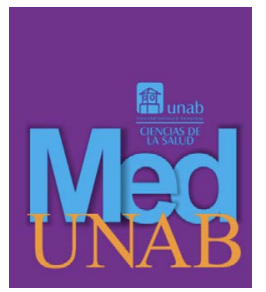

REVISTA DE LA FACULTAD

DE CIENCIAS DE LA SALUD

\title{
Malformación arteriovenosa de alto flujo en un adulto joven: reporte de caso
}

High flow arteriovenous malformation in a young adult: case report

Malformação arteriovenosa de alto fluxo em um adulto jovem: relato de caso

\section{Nefi Helaman Rodriguez-Londoño, Est. ${ }^{1}$ iD}

1. Médico interno, Universidad de Santander. Bucaramanga, Santander, Colombia.

Correspondencia. Nefi Helaman Rodriguez-Londoño. Carrera 48 numero 37-02, Urbanización el Paraíso, 3202891940. Email. nefirodriguez2404@gmail.com

\section{INFORMACIÓN DEL ARTÍCULO:}

Artículo recibido: 15 de octubre de 2019

Artículo aceptado: 13 de octubre de 2020

DOI: https://doi.org/10.29375/01237047.3785

Cómo citar. Rodriguez-Londoño NH. Malformación arteriovenosa de alto flujo en un adulto joven. MedUNAB. 2021;24(1): 72-79. doi: https://doi.org/10.29375/01237047.3785

\section{RESUMEN:}

Introducción. Las malformaciones arteriovenosas son lesiones relativamente raras e infrecuentes. Se caracterizan por presentar un aumento anormal en el número de vasos sanguíneos como consecuencia de un defecto en el desarrollo vascular. Constituyen un desafío diagnóstico y terapéutico para el médico tratante. Su incidencia es de alrededor el 1.5\% de la población general. Dentro de las opciones terapéuticas se incluye la embolización selectiva, la resección quirúrgica o ambas. El objetivo del presente artículo es reportar un caso de una patología poco frecuente y hacer una revisión literaria del tema para arrojar luz sobre su diagnóstico. Reporte de caso. Se presenta el caso de un paciente adulto joven que consulta por presentar una masa en glúteo derecho de 6 años de evolución. Esta es diagnosticada erróneamente como lipoma, por lo que se lleva al paciente a cirugía sin la realización de imágenes diagnósticas previas. En la cirugía, el paciente presenta choque hipovolémico. Posteriormente, se documenta la masa como malformación arteriovenosa profunda. Discusión. Es poco usual la ubicación de dichas malformaciones en los miembros inferiores, como en el paciente del actual caso. El diagnóstico de estas lesiones puede ser clínico, pero requiere del conocimiento o sospecha de esta entidad, ya que pueden ser lesiones clínicamente no visibles, lo que lleva a que pasen inadvertidas o se diagnostiquen de forma errónea. Conclusión. Aunque se trata de una patología 
poco frecuente, esta puede generar repercusiones clínicas, físicas, psicológicas y estéticas importantes, por lo que es indispensable realizar adecuados métodos por imágenes que permitan establecer su correcto diagnóstico y manejo.

Palabras claves:

Malformaciones vasculares; Embolización terapéutica; Angiografía; Neovascularización patológica; Choque hipovolémico.

\section{ABSTRACT}

Introduction. Arteriovenous malformations are relative rare and infrequent injuries. Their main characteristic is an abnormal increase in the number of blood vessels as a result of defective vascular development. They represent a diagnostic and therapeutic challenge for the treating physician. Their incidence in the general population is around $1.5 \%$. Some therapeutic options include selective embolization, surgical resection, or both. The purpose of this article is to report a case of an infrequent pathology and to perform a literature review on the topic to shed light on its diagnosis. Case report. The case involves a young adult patient who inquired about the presence of a mass in the right buttock with six years of evolution. It was erroneously diagnosed as a lipoma, as a result of which the patient was taken to surgery without performing preliminary diagnostic images. During surgery, the patient went into hypovolemic shock. Afterwards, the mass was documented as a profound arteriovenous malformation. Discussion. Such malformations are rarely found in the lower limbs, as in this case. These injuries may be clinically diagnosed, but knowledge or suspicion on the existence of this entity is required, because such injuries might not be clinically visible, which implies that they may go unnoticed or be erroneously diagnosed. Conclusion. Even though it is an infrequent pathology, it may have substantial clinical, physical, psychological and aesthetic implications, which implies that it is indispensable to perform adequate imaging-based procedures to enable its adequate diagnosis and management.

Keywords:

Vascular malformations; Therapeutic embolization; Angiography; Pathological neovascularization, Hypovolemic shock.

\section{RESUMO}

Introdução. As malformações arteriovenosas são lesões relativamente raras e infrequentes. São caracterizadas por apresentarem um aumento anormal do número de vasos sanguíneos como consequência de um defeito no desenvolvimento vascular. Constituem um desafio diagnóstico e terapêutico para o médico que trata. Sua incidência gira em torno de $1.5 \%$ da população geral. As opções de tratamento incluem embolização seletiva, ressecção cirúrgica ou ambas. O objetivo deste artigo é relatar um caso de patologia pouco frequente e fazer uma revisão bibliográfica sobre o assunto para lançar luz sobre seu diagnóstico. Relato de caso. Apresentamos o caso de um paciente adulto jovem que consultou por apresentar uma massa na nádega direita, de 6 anos de evolução. Isso é diagnosticado erroneamente como um lipoma, então o paciente é levado para cirurgia sem imagens diagnósticas prévias. Na cirurgia, o paciente apresenta um choque hipovolêmico. Posteriormente, a massa é documentada como uma malformação arteriovenosa profunda. Discussão. A localização dessas malformações nos membros inferiores é incomum, como no caso deste paciente. O diagnóstico dessas lesões pode ser clínico, mas requer conhecimento ou suspeita dessa entidade, pois podem ser lesões clinicamente invisíveis, o que as leva a passar despercebidas ou mal diagnosticadas. Conclusão. Embora seja uma patologia pouco frequente, pode gerar importantes repercussões clínicas, físicas, psicológicas e estéticas, pelo que é imprescindível a realização de métodos de imagem adequados para estabelecer seu correto diagnóstico e tratamento.

Palavras-chave:

Malformações vasculares; Embolização terapêutica; Angiografia; Neovascularização patológica; Choque hipovolêmico. 


\section{Introducción}

Las malformaciones arteriovenosas (MAV) son una anomalía relativamente rara, en particular las que ocurren en las extremidades inferiores (1). La teoría etiopatogénica de mayor consenso postula que estas se deben a un aumento en el número de vasos, debido a un defecto en el desarrollo vascular, especialmente en la angiogénesis (2). Datos epidemiológicos mundiales indican que la incidencia de las malformaciones vasculares congénitas es de $1.5 \%$, siendo aproximadamente $2 / 3$ partes de predominio venoso, afectando de forma similar a hombres y a mujeres, sin distinción entre grupos raciales (3). De estos casos solo el 1 al $2 \%$ de los pacientes presentan lesiones en los miembros inferiores (4).

Las malformaciones vasculares componen un espectro de lesiones amplio y heterogéneo que frecuentemente se presentan como un desafío diagnóstico y terapéutico para el médico tratante. El uso habitual de una nomenclatura poco clara y no actualizada ha llevado durante mucho tiempo a una considerable confusión (5). Las lesiones vasculares congénitas se dividen en hemangiomas y en malformaciones vasculares, dependiendo de sus características endoteliales. De acuerdo al canal vascular afectado, se dividen en lesiones de bajo flujo (venosas, linfáticas, capilares o una combinación de ellas) y de alto flujo (MAV y fístulas arteriovenosas). Las malformaciones vasculares de alto flujo se definen como derivaciones anormales entre arterias y venas sin un lecho capilar intermedio (6). Son infrecuentes y es importante reconocerlas, pues representan probablemente el grupo más grave de anomalías vasculares.

El 40-60\% de las MAV son visibles desde el nacimiento y el $30 \%$ se hacen visibles clínicamente durante la infancia. Pueden surgir en cualquier parte del cuerpo y, por lo tanto, tienen una amplia gama de manifestaciones clínicas. Sin embargo, en aproximadamente el $12 \%$ de la población afectada causan síntomas con variados grados de severidad. En un pequeño número de individuos de este grupo, los síntomas son lo suficientemente graves como para causar debilitamiento, o incluso la muerte. Anualmente, aproximadamente el 1\% de las personas que padecen de MAV mueren como consecuencia directa de estas lesiones (7).

La sintomatología de las MAV se relaciona con hipervascularización, presencia de shunts de diferente calibre, hipertensión venosa e isquemia tisular $(8,9)$. Se suelen expresar cuando son superficiales, como masas pulsátiles, generalmente con el aumento de la temperatura de la piel y frémito palpable. Pueden presentar sobrecrecimiento óseo y de partes blandas, además de asociarse a lesiones osteolíticas (10). En estadios avanzados, los pacientes desarrollan hipertensión venosa, hemorragia o isquemia/necrosis tisular cerca de la lesión. Las MAV de gran tamaño pueden asociarse con insuficiencia cardíaca congestiva debido a una mayor precarga cardíaca derecha. Estas MAV generalmente se vuelven sintomáticas durante la pubertad (11).

En la práctica, existen malformaciones vasculares con un curso clínico típico y que no requieren estudios por imágenes para su diagnóstico, mientras que otras son lesiones atípicas, profundas, cubiertas de piel normal o que comprometen estructuras vitales en las que el diagnóstico clínico es poco claro o insuficiente. Los estudios por imágenes son fundamentales para un diagnóstico preciso y para la decisión terapéutica adecuada (12).

La ecografía y el examen Doppler se realizan inicialmente para establecer las características del flujo sanguíneo y así poder determinar una impresión diagnóstica. La resonancia magnética permite valorar la anatomía vascular, la extensión de la lesión y su relación con las estructuras adyacentes (13). La arteriografía permite reconocer el detalle preciso de la angioarquitectura y hemodinamia de la malformación, que serán vitales para planear la terapia endovascular. La prueba de oro es la angiografía selectiva (14).

La decisión sobre el tratamiento invasivo debe ser tomada por un equipo interdisciplinario y se basa en los síntomas individuales y las posibles complicaciones del curso natural de la enfermedad (15). Debido a que las MAV de alto flujo no involucionan de forma espontánea, dentro de las opciones terapéuticas se incluyen la embolización selectiva, la resección quirúrgica o ambas (16). La embolización es eficaz en los casos en que el tratamiento quirúrgico no es posible. En combinación con la cirugía aumenta sus indicaciones, pues hace operables ciertos casos y permite intervenciones más seguras, sencillas y menos traumáticas. Se realiza de 24 a 72 horas previas a la cirugía con el objetivo de reducir la pérdida de sangre (17). La cirugía puede ser total o subtotal en función de la localización y del tamaño, siendo la curación definitiva cuando la extirpación es completa (18).

Esta es una patología infrecuente, por lo que se debe diagnosticar de manera temprana y adecuada para evitar complicaciones. Acá surge la importancia 
de divulgar este reporte de caso para enfatizar su etiología, sospecha clínica, correcta clasificación y adecuado tratamiento.

\section{Descripción del caso}

Paciente masculino de 20 años de edad residente del municipio de Barrancabermeja, Santander, estudiante universitario, previamente sano y sin antecedentes de importancia. Lo envían a la consulta de cirugía general por presentar masa en el cuadrante superior externo del glúteo derecho de consistencia dura, no móvil, dolorosa y pulsátil de 6 años de evolución, que había ido aumentando progresivamente de tamaño, limitándole la marcha. Ante sospecha clínica se le diagnostica "gran lipoma en glúteo derecho". Días después y sin la previa realización de ayudas imagenológicas diagnósticas lo intervienen quirúrgicamente con la intención de reseccionarle la lesión en su totalidad. Se encuentra una gran masa vascular de aproximadamente $10 x 12 \mathrm{~cm}$. En el intraoperatorio el paciente presenta un shock hipovolémico con compromiso hemodinámico, se suspende la intervención quirúrgica, y se realiza control con hemostasia a través de anudamiento y ligadura de múltiples vasos sanguíneos, suturas intradérmicas y externas. Horas después se le da egreso hospitalario y se le envía para valoración por parte de cirugía vascular. Nueve días después presenta dehiscencia de suturas externas con salida de escaso material serohemático y gran edema. Se le documenta posteriormente en una institución de mayor nivel de complejidad a través de eco Doppler y angioresonancia de pelvis contrastada: MAV profunda de glúteo derecho (figura 1).
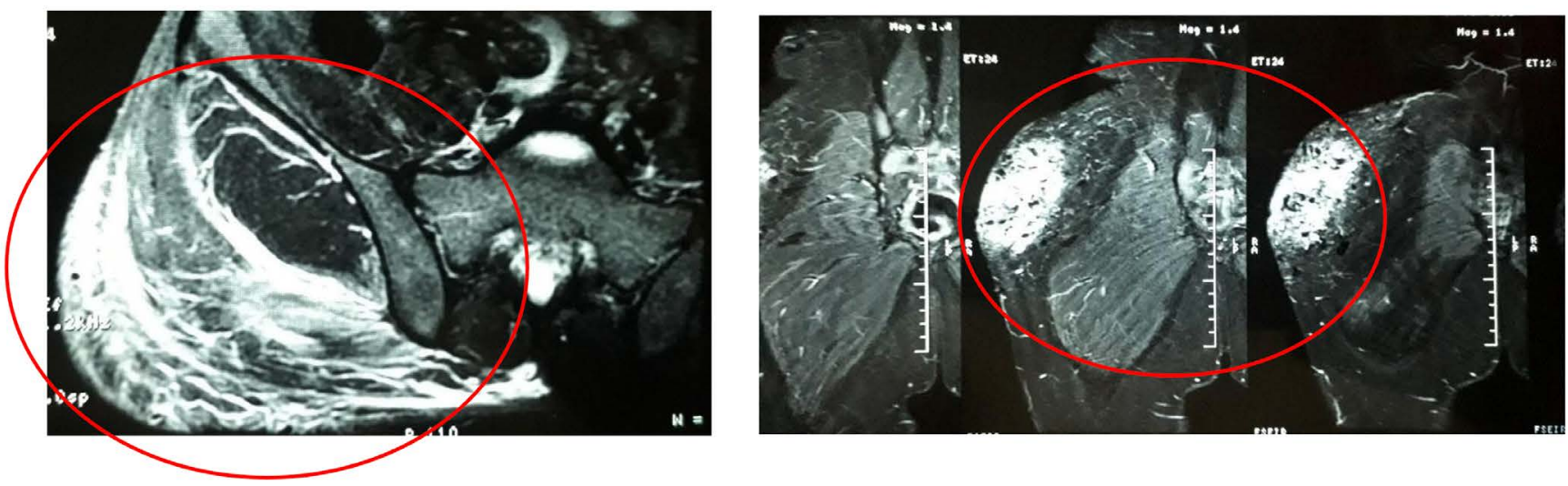

Figura 1. Angioresonancia de pelvis con contraste.

MAV con nicho confinado a la región subcutánea lateral de cadera derecha, alimentada por ramas de la arteria glútea superior y femoral profunda con drenaje a través de venas glúteas, femoral profunda y lumbar en la porción alta.

Fuente: Elaboración propia.

El paciente se presenta en junta médico-quirúrgica en donde se determina llevar a cabo la arteriografía de iliaca primitiva derecha con visualización selectiva de iliaca interna y arterias glúteas derechas y sus ramas para embolización endovascular (figura 2). Esta se lleva a cabo cinco meses después; en en el posoperatorio inmediato el paciente presenta tos seca, disnea, taquicardia sinusal, desaturación, hipoventilación derecha y trombocitopenia por consumo; documentada como tromboembolismo pulmonar periférico, el cual se le confirma a través de gammagrafía de ventilación y perfusión. En junta médica multidisciplinar y ante la mejoría clínica sintomática se le da de alta al paciente siete días después, con seguimiento por la consulta externa cada ocho días. Tres meses después, se realiza resección quirúrgica completa de la lesión, hasta la fascia muscular con colocación de colgajo a distancia en varios tiempos, e injerto parcial de piel por parte de cirugía de la mama y tumores, sin presentar complicaciones. Se envía muestra para estudio histopatológico donde se descarta malignidad y se confirma que la lesión corresponde a una malformación arteriovenosa (figura 3).

Finalmente, se realiza seguimiento médico especializado y ecográfico durante 3 meses, que son reportados como normales. 

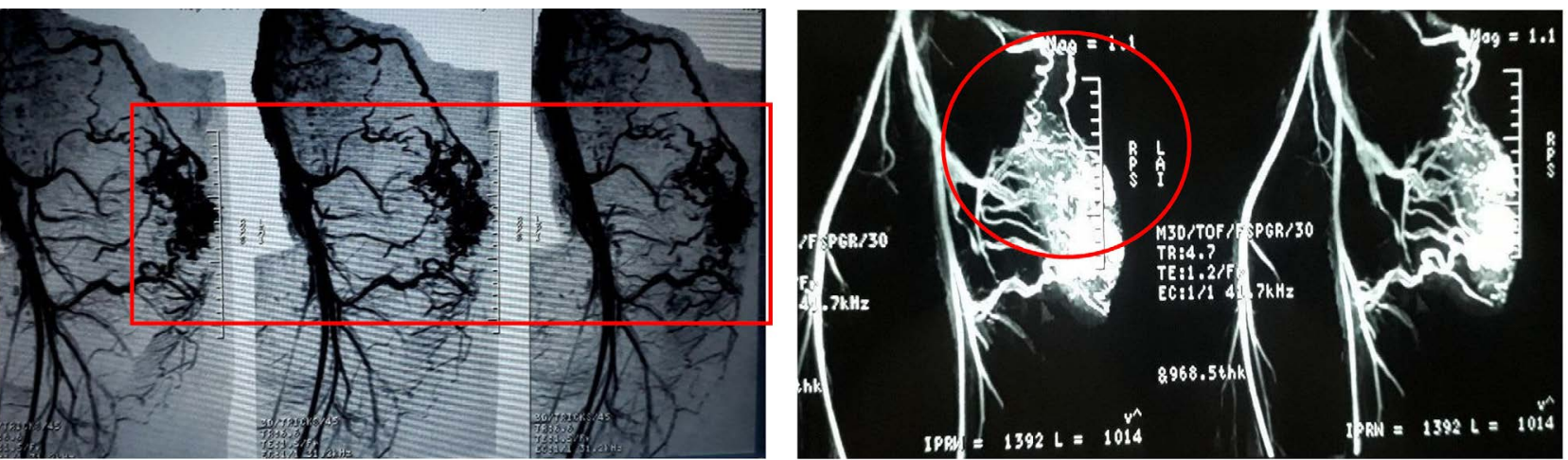

Figura 2. Arteriografía de ilíaca primitiva derecha.

Lesión glútea altamente vascularizada con múltiples vasos aferentes de gran tamaño sin opacificación venosa temprana.

Fuente: Elaboración propia.

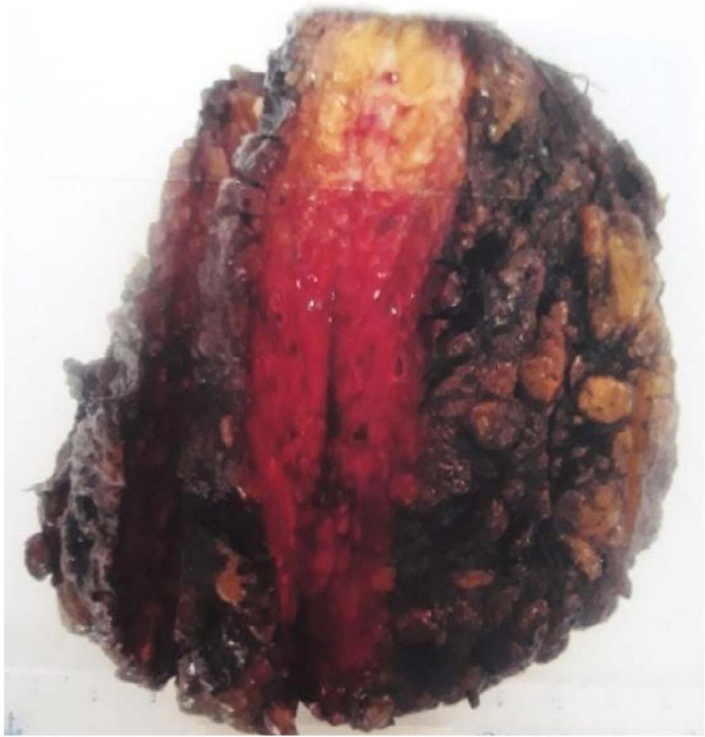

Figura 3. Espécimen patológico.

Descripción microscópica: Números vasos sanguíneos venosos, dilatados, tapizados por endotelio sin atipia, con trombos y material de cuerpo extraño en su interior (embolización previa) rodeados por un estroma congestivo, con ligero infiltrado inflamatorio linfocitario. No se observa malignidad, lesión correspondiente a malformación arteriovenosa

Fuente: Elaboración propia.

\section{Discusión}

Las malformaciones vasculares congénitas, incluidas las $\mathrm{MAV}$, son una patología poco común, razón por la cual siguen siendo una de las enfermedades más difíciles de diagnosticar y tratar terapéuticamente. Se producen en alrededor del 1 al 2.6\% de neonatos (19). Su origen genético ha sido admitido por algunos autores debido a que se establece una ausencia de apoptosis y desregulación de la diferenciación vascular. También está involucrada una mutación del gen RASA1. Sin embargo, ha habido casos de MAV secundarias a traumatismos, enfermedad vascular degenerativa y otras de origen iatrogénico que, al igual que con el paciente acá presentado, se diagnostican tarde porque las lesiones no siempre son visibles al nacer. Garzón et al. informan que las lesiones no están presentes en el $40-60 \%$ de los neonatos y solo se manifiestan en la adolescencia en alrededor del $30 \%$ de los casos (20). Enjorlas et al. habían encontrado esta misma variabilidad en la edad de revelación de MAV en su estudio (21).

En Colombia solo existe un caso similar reportado en Bogotá en el Hospital San José. Se trató de una paciente de 32 años de sexo femenino que consultó por presentar dolor al mover su miembro inferior izquierdo; presentaba una lesión que había crecido con el transcurso del tiempo. La paciente refería que dicha lesión la tenía desde la infancia, evidenciándose al principio como una pequeña mancha en la piel. Durante su adolescencia hubo un gran crecimiento y fue diagnosticada como hemangioma, con áreas de pigmentación y ulceración, sin auscultarse soplo en el sitio de lesión. Este caso se diferencia con el acá estudiado, del paciente de sexo masculino de Barrancabermeja porque este refirió que durante su infancia y adolescencia la lesión no era visible, versión que fue corroborada al interrogar a los padres. Sumado a esto, su alteración vascular sí era pulsátil. Estos casos tienen en común que las dos lesiones tuvieron un crecimiento exponencial durante la adolescencia con repercusiones funcionales, pues el dolor impedía la libre movilización del miembro afectado (22). En Santander no se encontraron datos estadísticos o información literaria que reportaran o describieran casos similares.

Las MAV de los miembros superiores se detectan en promedio a los 2 años de edad y se expresan con mayor frecuencia alrededor de los 11 años (23). El diagnóstico de MAV periféricas se puede determinar generalmente 
después de un examen clínico; al principio puede resultar difícil porque las manifestaciones son diversas, ya que pueden ser lesiones clínicamente no visibles, pueden estar ocultas en los músculos, se confunden con malformaciones capilares o, por el contrario, son lesiones significativas que pueden comprometer el pronóstico funcional o vital, siendo esta última una situación extremadamente rara ya que solo afecta al $1-2 \%$ de los pacientes, y se produce generalmente en casos de MAV proximal (24), debido a que las lesiones distales no muestran derivaciones suficientemente significativas para causar descompensación cardíaca (25).

Los síntomas se pueden describir utilizando el sistema de estadificación de Schobinger, el cual divide a estas malformaciones en cuatro estadios clínicos (Tabla 1).

Tabla 1. Estadios clínicos de las malformaciones arteriovenosas de alto flujo.

\begin{tabular}{cl}
$\begin{array}{c}\text { Estadio } \\
\text { clínico }\end{array}$ & \multicolumn{1}{c}{ Características } \\
\hline I & $\begin{array}{l}\text { Lesión cutánea tipo mácula, rosada-violácea, } \\
\text { caliente, generalmente asintomática. }\end{array}$ \\
\hline II & $\begin{array}{l}\text { Lesión clínicamente pulsátil, siendo evidente } \\
\text { la presencia de vasos tensos tortuosos, } \\
\text { ocasionalmente dolorosa. }\end{array}$ \\
& $\begin{array}{c}\text { Aparición de lesiones cutáneas distróficas, } \\
\text { ulceración, sangrado y dolor continuo. }\end{array}$ \\
III & $\begin{array}{l}\text { Se asocian falla cardíaca, infecciones que } \\
\text { no responden al tratamiento e isquemia } \\
\text { periférica. (25). }\end{array}$ \\
Fuente: Elaboración propia.
\end{tabular}

Ubicaciones como en las extremidades inferiores, como la del paciente acá estudiado, son muy raras. En este caso fue tipificada con estadio II dado por las características clínicas y sintomáticas que presentaba (26).

Otros autores encontraron que el flujo de MAV podría abruptamente acelerarse como resultado de los cambios hormonales, tales como durante pubertad, trauma, infecciones o cirugías. Este puede ser el caso del paciente que al momento de la consulta no presentaba antecedentes de importancia, considerado previamente sano y en donde la lesión se le corrige en la edad adulta. Pero con una evolución de seis años, tiempo en el cual se encontraba en el desarrollo puberal bajo el influjo de grandes cambios hormonales. Su diagnóstico inicial se realiza mediante una ecografía Doppler. Esta localiza los puntos de la fístula, evalúa las tasas de flujo y mide su resistencia (27); además, es más accesible y económica.

La angio-tomografía contrastada que se le realizó al paciente ofrece, en comparación con la ecografía Doppler, una mejor visualización de las estructuras vasculares de la MAV, su extensión esquelética o visceral. En nuestro medio, la arteriografía contrastada es el gold standard para explorar las MAV porque caracteriza mejor las arterias nutricias y las venas drenaje de la lesión. Por otra parte, Johnson et al. tienen recomendado, para comenzar con el diagnóstico, realizar en primera instancia una RMN, debido a que el eco Doppler no puede explorar lesiones profundas y cercanas a estructuras óseas o que contengan aire (28). Pero esto no es siempre accesible en Colombia debido a los costos y trámites administrativos. Por lo tanto, al paciente se le confirmó el diagnóstico basándose en primera instancia en el examen de eco Doppler, complementando con la angiografía tomografía contrastada y la arteriografía.

\section{Conclusión}

Las malformaciones vasculares son una entidad patológica bastante rara, poco frecuente pero importante, dadas las repercusiones clínicas (en algunos casos con elevada morbimortalidad), físicas, psicológicas, estéticas, y que para su tratamiento más idóneo requiere del trabajo mancomunado de un equipo multidisciplinario. Para ello es indispensable realizar un diagnóstico y una clasificación adecuada, soportados en métodos por imágenes, antes de tomar cualquier iniciativa terapéutica.

Actualmente las técnicas intervencionistas ofrecen una amplia gama de alternativas terapéuticas de primera línea, seguras y efectivas para el manejo de este tipo de alteraciones que conllevan a mejorar la calidad de vida de estos pacientes.

\section{Consideraciones éticas}

Se obtuvo el consentimiento informado por escrito del paciente para la publicación de este informe y las imágenes que lo acompañan.

\section{Conflicto de interés}

El autor declara no tener ningún conflicto de interés. 


\section{Agradecimientos}

En memoria: De mi amado sobrino Nefi Sebastián, que, en su corto tiempo, vino alegrar nuestras vidas y su partida deja una gran tristeza en nuestros corazones, su recuerdo perdurará para siempre entre nosotros.

\section{Referencias}

1. Danielson L, Anderson J, Nykamp M, Remund T, Kelly P. Treatment of a Massive Left Femoral Arteriovenous Malformation Using an Innovative Modular Hybrid Bifurcated Stent Graft System. Annals of Vascular Surgery. 2014;28(4):1031.e151031.e20. https://doi.org/10.1016/j.avsg.2013.07.023

2. El-Fiki M. Angiogenesis Detection in Cerebral Arteriovenous Malformations: Mediators and Gene Expression, and Treatment Hopes for the Future. World Neurosurgery. 2012;78(5):422-424. https://doi. org/10.1016/j.wneu.2012.04.024

3. Redondo P, Aguado L. Actualización en malformaciones venosas. Anales del Sistema Sanitario de Navarra. 2010;33(3). https://doi.org/10.4321/ S1137-66272010000400006

4. Churojana A, Khumtong R, Songsaeng D, Chongkolwatana C, Suthipongchai S. LifeThreatening Arteriovenous Malformation of the Maxillomandibular Region and Treatment Outcomes. Interventional Neuroradiology. 2012;18(1):49-59. https://doi.org/10.1177/159101991201800107

5. Lojo Rocamonde I, Moro Mayor A, Barreiro Veiguela J. Malformaciones vasculares periféricas. Diagnóstico y tratamiento. Angiología. 2012;64(4): 147-154. https://doi.org/10.1016/j.angio.2012.01.005

6. Deng W, Huang D, Chen S, Zhang X, Li X, Li J et al. Management of High-Flow Arteriovenous Malformation in the Maxillofacial Region. Journal of Craniofacial Surgery. 2010;21(3):916-919. https:// doi.org/10.1097/SCS.0b013e3181d880fd

7. Naouri M, Lorette G, Barbier C, Zakine G, Herbreteau D. Malformations artérioveineuses. La Presse Médicale. 2010;39(4):465-470. https://doi. org/10.1016/j.lpm.2010.01.004

8. Liu A, Mulliken J, Zurakowski D, Fishman S, Greene A. Extracranial Arteriovenous Malformations: Natural Progression and Recurrenceafter Treatment. Plastic and Reconstructive Surgery. 2010;125(4):1185-1194. https://doi.org/10.1097/PRS.0b013e3181d18070

9. Dunham G, Ingraham C, Maki J, Vaidya S. Finding the Nidus: Detection and Workup of Non-Central Nervous System Arteriovenous Malformations. RadioGraphics. 2016;36(3):891-903. https://doi. org/10.1148/rg.2016150177
10. Garzon M, Huang J, Enjolras O, Frieden I. Vascular malformations. Journal of the American Academy of Dermatology. 2007;56(3):353-370. https://doi. org/10.1016/j.jaad.2006.05.069

11. Ballah D, Cahill A, Fontalvo L, Yan A, Treat J, Low $\mathrm{D}$ et al. Vascular Anomalies: What They Are, How to Diagnose Them, and How to Treat Them. Current Problems in Diagnostic Radiology. 2011;40(6):233247. https://doi.org/10.1067/j.cpradiol.2011.04.001

12. Legiehn G, Heran M. Venous Malformations: Classification, Development, Diagnosis, and Interventional Radiologic Management. Radiologic Clinics of North America. 2008;46(3):545-597. https://doi.org/10.1016/j.rcl.2008.02.008

13. Sierre S, Teplisky D, Lipsich J. Malformaciones vasculares: actualización en diagnóstico por imágenes y tratamiento. Archivos Argentinos de Pediatría. 2016;114(2):167-176. [Internet]. 2016 [cited 7 April 2021]; Available from: https://www.sap.org. ar/docs/publicaciones/primero/2016/Act_Sierre anticipo 9-3-16.pdf

14. Dubois J, Alison M. Vascular anomalies: what a radiologist needs to know. Pediatric Radiology. 2010;40(6):895-905. https://doi.org/10.1007/s00247010-1621-y

15. Flors L, Leiva-Salinas C, Maged I, Norton P, Matsumoto A, Angle $\mathrm{J}$ et al. MR Imaging of Soft-Tissue Vascular Malformations: Diagnosis, Classification, and Therapy Follow-up. RadioGraphics. 2011;31(5):1321-1340. https://doi. org/10.1148/rg.315105213

16. Alomari A, Dubois J. Interventional Management of Vascular Malformations. Techniques in Vascular and Interventional Radiology. 2011;14(1):22-31. https:// doi.org/10.1053/j.tvir.2010.07.006

17. Grigg, C., Anderson, D. and Earnshaw, J. Diagnosis and Treatment of Hereditary Hemorrhagic Telangiectasia. The ochsner Journal, 17(2), pp.157161. [Internet]. 2017 [cited 7 April 2021]; Available from: https://www.ncbi.nlm.nih.gov/pmc/articles/ PMC5472075/.

18. Górriz Gómez E, Carreira J. Tratamiento percutáneo de las malformaciones vasculares periféricas con una mezcla de polidocanol y CO2. Experiencia inicial. Radiología. 2008;50(5):424-429. https://doi. org/10.1016/S0033-8338(08)76058-2

19. Müller-WilleR, WildgruberM, SadickM,Wohlgemuth W. Vascular Anomalies (Part II): Interventional Therapy of Peripheral Vascular Malformations. RöFo - Fortschritte auf dem Gebiet der Röntgenstrahlen und der bildgebenden Verfahren. 2018;190(10):927-937. https://doi.org/10.1055/s-0044-101266

20. Husain T, Garrido D, Aziz-Sultan A, Tang J, Salgado C. Use of Immediate Dermal Fat Graft for Scalp 
Contour Defect Following Resection of Arteriovenous Malformation. Journal of Craniofacial Surgery. 2012;23(5):e380-e384. https://doi.org/10.1097/ SCS.0b013e3182587b54

21. Vanwijck, R. and Dégardin-Capon, N. Les malformations artérioveineuses: aspects cliniques et volution. Annales de Chirurgie Plastique Esthétique, 51(4-5), pp.440-446. https://doi.org/10.1016/j. anplas.2006.07.011

22. Riascos R, Martinez S, Molano E, Fuentes J. Anomalías vasculares de tejidos blandos: Dilema en terminología y enfoque diagnóstico. Revisión de la literatura y presentación de un caso. Repertorio de Medicina y Cirugía. 2004;13(2):67-71. https://doi. org/10.31260/RepertMedCir.v13.n2.2004.345

23. Fernández R L, Boettiger $\mathrm{B} \mathrm{P}$, Bahamonde $\mathrm{S} \mathrm{H}$. Resección quirúrgica de malformación arteriovenosa de alto flujo en ángulo nasogeniano con embolización preoperatoria: Reporte de un caso. Revista de otorrinolaringología y cirugía de cabeza y cuello [Internet]. 2013 [cited 7 April 2021];73(1):63-67. Available from: https://scielo.conicyt.cl/pdf/orl/ v73n1/art10.pdf

24. Wu I, Orbach D. Neurointerventional Management of High-Flow Vascular Malformations of the Head and Neck. Neuroimaging Clinics of North America.
2009;19(2):219-240. https://doi.org/10.1016/j. nic.2009.01.005

25. Burrows $\mathrm{P}$, Mulliken J, Fishman S, Klement G, Folkman J. Pharmacological Treatment of a Diffuse Arteriovenous Malformation of the Upper Extremity in a Child. Journal of Craniofacial Surgery. 2009;20(Suppl 1):597-602. https://doi.org/10.1097/ SCS.0b013e3181927fle.

26. De Miguel, R., López-Gutierrez, J. and Boixeda, P. Arteriovenous Malformations: A Diagnostic and Therapeutic Challenge. Actas Dermo-Sifiliográficas (English Edition), 105(4), pp.347-358. https://doi. org/10.1016/j.adengl.2013.04.026

27. Toker M, Eren E, Akbayrak H, Numan F, Güler M, Balkanay $\mathrm{M}$ et al. Combined approach to a peripheral congenital arteriovenous malformation: surgery and embolization. Heart and Vessels. 2006;21(2):127130. https://doi.org/10.1007/s00380-005-0842-8.

28. Johnson J, Cogswell P, McKusick M, Binkovitz L, Riederer S, Young P. Pretreatment imaging of peripheral vascular malformations. Journal of Vascular Diagnostics. 2014;:121. https://doi.org/10.2147/JVD. $\underline{\text { S66467. }}$. 